\title{
Molecular docking of active compounds from Phoenix pusilla root extract against antidiabetic and anti-inflammatory drug targets
}

\author{
Vijayabharathi Saravanan ${ }^{1}$, Anuradha Venkatraman ${ }^{2 *}$, Habeeb Shaik Mohideen ${ }^{3}$ \\ ${ }^{1}$ Department of Biotechnology, FSH, SRM University, Chengalpattu, India. \\ ${ }^{2}$ Department of Biochemistry, Mohamed Sathak College of Arts and Science, Chennai, India. \\ ${ }^{3}$ Department of Genetic Engineering, School of Bioengineering, SRM Institute Science and Technology, Kattankulathur, Tamil Nadu, India.
}

\begin{tabular}{l}
\hline ARTICLE INFO \\
\hline Article history: \\
Received on: January 27, 2021 \\
Accepted on: March 12, 2021 \\
Available online: September 20, 2021 \\
\hline
\end{tabular}

Key words:

Phoenix pusilla, docking, igemdock, vanillic acid, alpha

amylase, alpha glucosidase

\begin{abstract}
Phoenix pusilla is a stemless plant found in India and Srilanka belonging to arecaceae family, commonly called as small date palm. Small date palm found to have folklore medicinal value but it is underutilized. Hence, in this present study, antidiabetic and anti-inflammatory activity of active compounds from P. pusilla root ethanol extract were assessed using in silico tools. Two antidiabetic and two anti-inflammatory protein targets were chosen for the docking study. The lead molecules were also assessed for drug likeliness properties using molinspiration. Eight ligands were selected from the results of GC-MS chromatogram of P. pusilla root ethanol extract. Docking was carried using igemdock and autodock software. From igemdock results, it was observed that among the eight compounds chosen, vanillic acid showed high binding efficiency with all the four target proteins. The binding energy of vanillic acid observed against alpha amylase and alpha glucosidase were -65.52 and -61.63 , respectively. Similarly, the binding energy against Interleukin 6 and tumour necrosis factor alpha were -62.116 and -78.0683 , respectively. Phoenix pusilla root could be utilized as a source for vanillic acid and also as an antidiabetic and anti-inflammatory agent.
\end{abstract}

\section{INTRODUCTION}

Phoenix pusilla (PP) is a shrub commonly grows up to $3 \mathrm{~m}$. It consists of pinnately arranged leaves and per year about 15-17 leaves are produced. Leaves terminus have sharp end which resembles thorns. Propagation is through seed, flowering season is during November and ends in January. Fruit season is in July and August. Roots are fibrous which fix the plant to the soil [1]. In India, P. pusilla is commonly seen in coromandel coast of Tamil Nadu (Chengalpattu, Thanjavur, Salem, South Arcot, Trichy, and Tirunelveli) and in south Kerala [2]. PP is one among the 250 plants listed as most important Indian Ayurvedic plants [3]. The root of PP was used in the ayurvedic formulation, which is used to treat heart disease [4].

\footnotetext{
*Corresponding Author

Anuradha Venkatraman, Department of Biochemistry, Mohamed Sathak College of Arts and Science, Chennai, India. E-mail: vanuradha2712@gmail.com
}

In silico analysis of proteins is an effective alternative research method, to carry out at the molecular level. The major branch of molecular modelling and docking has been predominantly applied in the field of drug designing and discovery. Molecular docking approach helps to predict the favorable binding orientation of single or multiple candidate molecules to their target proteins involved in causing diseases and disorders. A protein-ligand docking program consists of two essential components, sampling and scoring. Sampling refers to the generation of putative ligand binding orientations/conformations near a binding site of a protein and Scoring is the prediction of the binding tightness for individual ligand orientations/conformations with a physical or empirical energy function [5]. The top conformation is predicted as the binding mode. The three key ingredients in the proteinligand docking are representation of the system, conformational space search, and ranking of potential solutions.

The binding energy of the target proteins and small molecules in docking are solely dependent on the scoring functions. The free energy associated with docking is computed to determine the docking score [6]. Various online docking software available 
includes: click docking, TarFis dock, INV DOCK, Autodock, Glide, GOLD, LigandScout, FlexX, OEdocking, igemdock, Molegro Virtual Docker, etc. These in silico tools plays vital role in drug designing [7].

\section{MATERIALS AND METHODS}

\subsection{Target Protein Selection}

Anti-diabetic and anti-inflammatory protein targets were chosen for the docking study. Target proteins were downloaded in .pdb format from www.rcsb.org and used for docking studies.

\subsubsection{Alpha-amylase}

Human pancreatic alpha - amylase enzyme is a single polypeptide chain composed of 496 amino acids encoded on chromosome 1. The enzyme has three structural domains. Residues 1-99, 169-404 comprised the largest domain known as domain A. This largest domain consists of an eight-stranded parallel $\beta$ barrel surrounded by a concentric cylinder of $\alpha$-helical segments. On one end of this largest domain, the active site residues of the protein Asp 197, Glu 233, and Asp 300 are found. In addition, a bound chloride ion was also found in the vicinity (Arg 195, Asn 298, and Arg 337) of the protein domain. Next to domain A is the smallest domain B comprising residues 100-168. This domain possesses a calcium binding site (Asn 100, Arg 158, Asp 167, and His 201) toward domain A. The third domain is domain C (residues 405-496) made up of antiparallel beta structure. This small domain is loosely associated with domains A and B [8]. Both salivary and pancreatic alpha amylase are highly homologous in nature. Generally, salivary alpha amylase initiates partial digestion which breaks down polymeric starch into shorter oligomers. During the alpha amylase enzyme hydrolysis, $\alpha-1,4$ glucosidiclikages is broken down in two steps. In the first step, an intermediate glycosyl enzyme is formed along with a catalytic nucleophile (Asp 197) and acid/base catalyst (Glu 233). In the second step, the glycosyl intermediate formed is hydrolyzed releasing glucose monomers [9].

\subsubsection{Alpha glucosidase}

Alpha glucosidase is an exo-type carbohydrase present widely in microorganisms, plants, and animal tissues. The key enzymes in the carbohydrate digestion pathway are alpha amylases and alpha glucosidases. Alpha amylases act on long chain carbohydrates, whereas alpha glucosidases act on shorter starch chains and disaccharides to produce glucose. The glucose thus released will be absorbed in the blood. This leads to increased post-prandial blood glucose levels. Inhibiting these enzymes will reduce post-prandial glucose levels in Type 2 diabetes. Similar to alpha amylases, the structure of alpha glucosidases is also characterized by a barrel consisting of eight beta strands and alpha helices. It is also noted that alpha-amylase has both Asp and Glu residues in the active site and belongs to glycoside hydrolase family 13 whereas alpha glucosidase has only Asp residues in the active site and belongs to glycoside hydrolase family 31 [10].

\subsubsection{Interleukin-6}

The cytokine interleukin 6 performs diverse stimulatory functions in normal physiology and disease conditions. The structure of IL-6 has four helices A, B, C, and D. Among the four helices, A and B run in same direction whereas $\mathrm{C}$ and $\mathrm{D}$ run in opposite direction. A long loop like structures between the four helices holds them together. The first helix A is positioned from Ser 21 to Ala 45 and is associated with helix B by 25 amino acid loop. The disulphide bonds are seen between Cys 44 and Cys 50. The hydrophobic side chains are observed in amino acids Leu 62, Leu 64, Phe 65, and Met 67. Helix B runs between Glu 80 to Gln 102. The average $\varphi$ and $\psi$ torsion angles lies between $-63.8^{\circ}$ and $-39.5^{\circ}$, respectively. The bend observed in this helix regin results in a break in the alpoha helical $\mathrm{H}$ bonding pattern. The loop that connects $\mathrm{B}$ and $\mathrm{C}$ helix region lies betweeeen Asn 103 to Ser 108. Helix C region is formed between Glu 109 to Lys 129. The hydrophobic side chains are observed in Ile 136, Pro 139, and Leu 133. Pro 141 ro Gln 152 forms the three turns of the helix. Gln 156 to Arg 182 forms the $d$ helix region. Trp 157 is the only trptophan seen in the $\mathrm{N}$ termainal region. Gln 183 and Met 184 shows high $\mathrm{B}$ factor $35.9 \mathrm{~A}^{\circ}[11]$.

\subsubsection{Tumor necrosis factor alpha $(T N F-\alpha)$}

A protein hormone secreted by macrophages is TNF- $\alpha$. The secretion of TNF- $\alpha$ is mainly due to various inflammatory agents especially endotoxin. The monomer of TNF- $\alpha$ is 17,350 da forming an elongated, antiparallel beta pleated sheet with "jellyroll" topology. Three monomers associate and form a beell shaped trimeric structure. TNF- $\alpha$ is encoded in Major Histocompatibility Complex and is found to act synergestically with IL-1, IL-2, and $\gamma$ - interferon. The mature TNF- $\alpha$ is formed due to the occurance of a cleavage at 76 residue pepide from the prohormone. An intramolecular disulphide bridge is observd in the mature protein [12]. Compared to monomeric from the trimeric, TNF- $\alpha$ binds to the cell surface receptors with high affinity leading to the aggregation of receptors thereby inturn trigerring cellular response.

\subsection{Ligand Selection}

From the results of GC-MS analysis of PP (PARC/2017/3396) root ethanol extract, the bioactive compounds individually screened from review of literature for exhibiting anti-inflammatory and antidiabetic property and finally eight compounds were chosen for docking study.

\subsection{Target Protein Preparation}

The three-dimensional structure of target proteins alpha-amylase, alpha glucosidase Interleukin 6 , and TNF- $\alpha$ were downloaded from Research Collaboratory for Structural Bioinformatics (RCSB) protein databank. The inhibitors and ligands bound to the target protein were removed by releasing the atom coordinates. All the water molecules and Hetero atoms were deleted. Hydrogen bonds were added and gasteiger charges were assigned to the target proteins before converting them into pdbqt format [13].

\subsection{Ligand Preparation}

Hexanoic acid (Pubchem id:8892), tetradeca methyl cycloheptasiloxane (Pubchem id:7874), vanillic acid (Pubchem id:8468), tetradecanoic acid (Pubchem id:11005), n-hexadecanoic acid (Pubchem id:985), 9, 12-octadecadienoic acid (Pubchem id:3931), oleic acid (Pubchem id:445639), and 11-octadecenal (Pubchem id:5283382) were downloaded from PubChem in spatial 
data file format. The physicochemical properties of the ligands and drug-likeness were checked by using molinspiration [14] before proceeding with docking. Molecular weight and hydrogen bonding interactions are the major structural determinants of protein ligand binding. These ligand structures were converted into .pdb format using discovery studio. Later these were converted to the required pdbqt format in autodock for docking.

\subsection{Software Used}

For visualizing and modifying receptor-ligand structures, chemsketch, pymol, and discovery Studio were used. Pymol is an interactive protein visualization and editing tool. To initially estimate the pharmacological interventions between the receptor protein and ligand molecules, igemdock software was used. igemdock is a standalone software suite used for docking and virtual screening of receptor-ligand complex. The software provides interactive interfaces for both receptor and ligand compounds [15]. For the docking process, each pre-processed target proteins and library of ligands eight compounds were uploaded in igemdock. The output path was set and docking was started. The ligand fitness was calculated once docking is completed.

From the results of igemdocking, compounds showing high affinity towards target proteins were chosen and docked again in Autodock software (Scripps Research Institute, San Diego, USA). Autodock is a computational docking program used in this study. This method is based on an empirical free energy force field and logical grid array search method. It used grid dimensions for calculating binding energy of trail conformations [16]. The graphical user interfaces used in this software are MGL, ADT, and PMV.

\section{RESULTS AND DISCUSSION}

Four protein targets were chosen according to diversity of binding site properties. Crystal structures of alpha amylase (PDB code: 4GQR, resolution 1.20 А), alpha glucosidase (PDB code: 5NN5, resolution $2.00 \AA$ ), interleukin 6 (PDB code: $1 \mathrm{ALU}$, resolution $1.90 \AA$ ), and TNF- $\alpha$ (PDB code: 1 TNF, resolution $2.60 \AA$ ) were retrieved from the RCSB protein data bank and visualized using University of California, San Francisco chimera.

The three-dimensional structure of chosen eight ligands (Fig. 1) was downloaded from pubchem database in sdf format and converted to pdb format for binding with the target proteins. Also, the drug likeness score of the ligands was calculated by considering molecular weight, number of heavy atoms, number of hydrogen donor, number of hydrogen acceptor and number of violations, number of rotatable bonds. All the eight ligands chosen satisfied drug likeness property (Table 1).

In the present study, the molecular docking was carried out with two docking softwares. First, to understand the pharmacological interventions, the selected eight ligand compounds were docked against all antidiabetic and anti-inflammatory target proteins in igemdock software.

From igemdock results, it was observed that among the eight compounds chosen, vanillic acid showed high binding efficiency with all the four target proteins. The binding energy of vanillic acid observed against alpha amylase and alpha glucosidase were
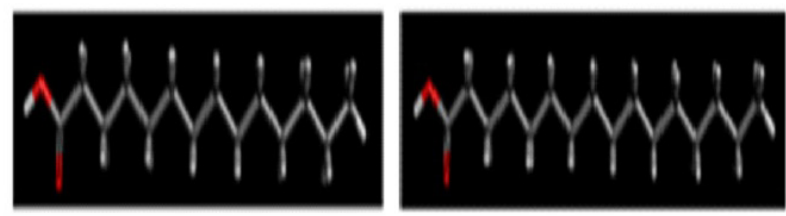

Hexanoic acid

11-Octadecanal
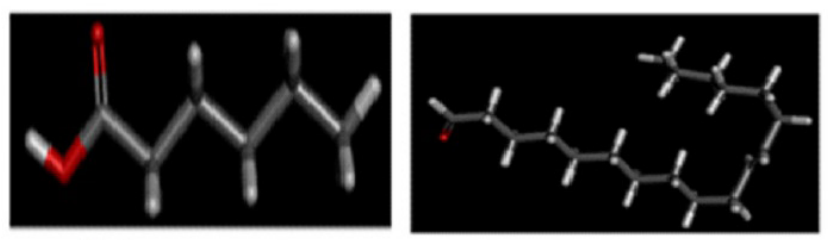

9,12-Octadecadienoic acid

Tetradecamethyl cyclohepta siloxane
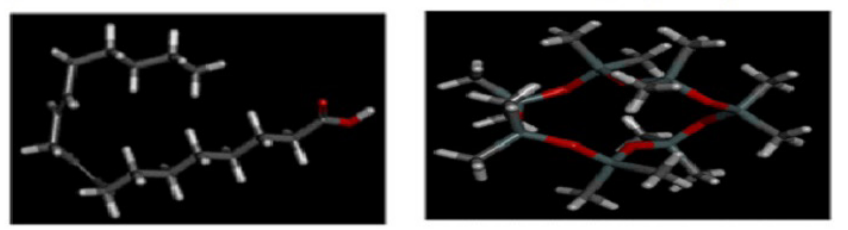

Vanillic acid

Oleic acid
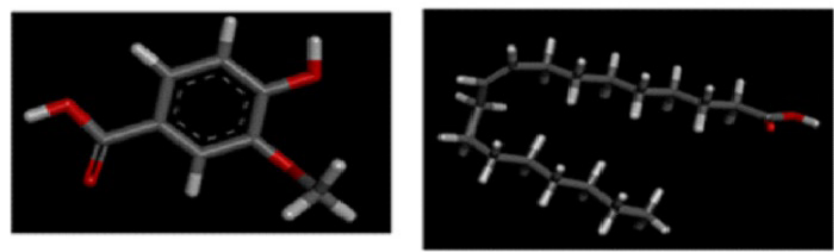

Figure 1. Ligands chosen for in silico analysis.

-65.52 and -61.63 , respectively. Similarly, the binding energy against Interleukin 6 and TNF- $\alpha$ were -62.116 and -78.0683 , respectively. The docking score of remaining seven compounds are shown in Table 2. Among the ligands chosen for the study, tetradecamethyl cycloheptasiloxane showed least binding energy which may be due to lack of activity which was checked.

Since vanillic acid showed high binding efficacy with all the target proteins in igemdocking, molecular docking of vanillic acid with alpha amylase, alpha glucosidase, interleukin 6 , and TNF- $\alpha$ was performed again using Autodock, a computational standalone software for predicting $\mathrm{H}$ bond interactions, $\mathrm{C}-\mathrm{H}$ bond interaction, pi-cation, and pi-anion interactions and unfavorable donors (Fig. 2).

In silico analysis of antidiabetic and anti-inflammatory activity of the selected ligands against the targets by docking revealed that vanillic acid was showing strong binding against the targets. Among the antidiabetic targets, vanillic acid was effective in binding with alpha amylase than to alpha glucosidase. Therefore, vanillic acid found in Phoenix pusilla ethanol extract was an effective alpha amylase inhibitor. Similarly, the anti-inflammatory docking study showed that vanillic acid was showing highest binding energy with TNF- $\alpha$. 
Table 1: Drug likeness of the compounds chosen for docking analysis.

\begin{tabular}{|c|c|c|c|c|c|c|}
\hline Compounds & Mol formula & Mol weight & H bond donor & H bond acceptor & $\log p$ & Molar refractivity \\
\hline 11-Octadecenal & $\mathrm{C} 18 \mathrm{H} 34 \mathrm{O}$ & 266.5 & 0 & 1 & 6.22 & 85.51 \\
\hline Vanillic acid & $\mathrm{C} 8 \mathrm{H} 8 \mathrm{O} 4$ & 168.15 & 2 & 4 & 1.09 & 41.61 \\
\hline 9,12-Octadecadienoic acid & $\mathrm{C} 18 \mathrm{H} 32 \mathrm{O} 2$ & 280.4 & 1 & 2 & 5.88 & 86.99 \\
\hline Oleic acid & $\mathrm{C} 18 \mathrm{H} 34 \mathrm{O} 2$ & 282.5 & 1 & 2 & 6.1 & 87.08 \\
\hline Tetradecanoic acid & $\mathrm{C} 14 \mathrm{H} 28 \mathrm{O} 2$ & 228.37 & 1 & 2 & 4.77 & 68.71 \\
\hline Hexadecanoic acid & $\mathrm{C} 16 \mathrm{H} 32 \mathrm{O} 2$ & 256.42 & 1 & 2 & 5.55 & 77.94 \\
\hline Hexanoic acid & $\mathrm{C} 6 \mathrm{H} 12 \mathrm{O} 2$ & 116.16 & 1 & 2 & 1.65 & 31.77 \\
\hline $\begin{array}{l}\text { Tetradecamethyl cyclo hepta } \\
\text { siloxane }\end{array}$ & $\mathrm{C} 14 \mathrm{H} 42 \mathrm{O} 7 \mathrm{Si} 7$ & 519.07 & 0 & 7 & 1.82 & 142.08 \\
\hline
\end{tabular}

Mol formula $=$ Molecular formula; Mol weight $=$ Molecular weight.

Table 2: Total Binding energy of docked ligands obtained by igemdock.

\begin{tabular}{|c|c|c|c|c|}
\hline Compound & Alpha glucosidase & Alpha amylase & TNF- $\alpha$ & Interleukin 6 \\
\hline 11-Octadecenal & -50.5456 & -59.5806 & -75.0541 & -50.6717 \\
\hline Vanillic acid & -61.6314 & -65.5221 & -78.0683 & -62.116 \\
\hline Oleic acid & -55.4653 & -59.336 & -72.8441 & -58.9936 \\
\hline 9,12-Octadecadienoic acid & -48.2468 & -57.845 & -71.4093 & -49.4471 \\
\hline Tetradecanoic acid & -56.1171 & -52.4471 & -70.0806 & -57.0538 \\
\hline Hexadecanoic acid & -48.8179 & -46.5324 & -68.5167 & -58.0289 \\
\hline Hexanoic acid & -44.493 & -44.8306 & -60.0532 & -47.4896 \\
\hline Tetra decamethyl cyclo heptasiloxane & -33.7981 & -17.029 & -41.0896 & -20.7921 \\
\hline
\end{tabular}
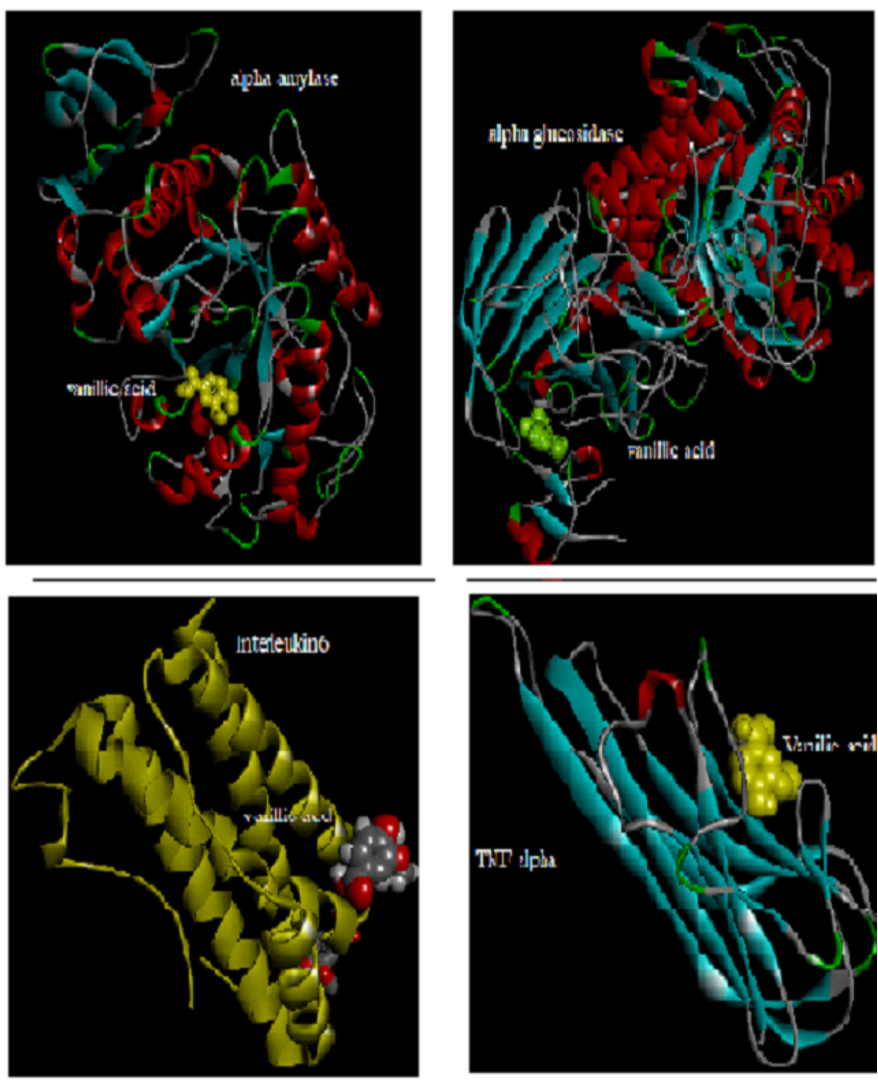

Figure 2. Molecular docking of vanillic acid with antidiabetic and antiinflammatory target proteins.
Chang et al. [17] studied the potential of vanillic acid as antidiabetic compound in high fed diet male Sprague-Dawley rats. Results obtained revealed that the Vanillic acid was found having effect of lowering blood glucose, triglycerides, low density lipoproteincholesterol, cyclooxygenase-2, and monocyte chemoattractant protein-1 expression. It was also found that the vanillic acid improved the signalling pathway of glucose uptake.

Asif et al. [18] reported that aqueous extract of St. Paul's wort plant showed antidiabetic effect was may be due to the presence of active compounds they identified by high performance liquid chromatography (HPLC) analysis of the aqueous extract plant. Vanillic acid was one of the compounds that was found in the plant extract. Also reported that the presence of vanillic acid along with other compounds identified by HPLC may be responsible for antidiabetic effect observed.

Four different seeds varieties of date palm were evaluated for anti-inflammatory activity by in vitro and in vivo method. It was found that the methanol seed extracts showed effective membrane stabilization property, protein denaturation action, and nitric oxide scavenging activity. Similarly, animal study showed that the extracts were effective in anti-inflammation activity and the phenol content analyzed showed that the phenols in the seeds were responsible for the effect. Among the phenols, vanillic acid was also reported to present in the seeds and it may be responsible for anti-inflammatory activity observed [19].

Review report by Oberoi and Kansra [20] on economic burden due to diabetes revealed that cost rise was seen. Diabetes prevalence in India was due to lack of proper diet, regular physical activities, 
regular check-up, and regular intake of drugs. They found that such self-care activities like diet and exercise was poor among the studied population of India.

Most of the in vivo study reported vanillic acid as a candidate of antidiabetic and anti-inflammatory activity. In the present study also, in silico analysis showed that vanillic acid was having antidiabetic and anti-inflammatory activity. So based on the in silico study and as reported in many research findings, vanillic acid might played vital role in antidiabetic and anti-inflammatory activity observed in the present study.

\section{CONCLUSION}

Vanillic acid is known for its antidiabetic effect by various mechanism and as the vanillic acid found present in PP root, it could be the choice for the source of vanillic acid as well as could be used for treating diabetes. Based on the present research work findings, it was identified that $P$. pusilla root was effective in hypoglycemic action as well as in regulating the secondary complications of diabetes. Therefore, it can be utilized as an important pharmaceutical agent in the treatment and management of diabetes complications.

\section{CONFLICT OF INTEREST}

Authors declare that they do not have any conflicts of interest.

\section{FUNDING}

No funding was received for this research work.

\section{AUTHOR CONTRIBUTIONS}

All authors made substantial contributions to conception and design, acquisition of data, or analysis and interpretation of data; took part in drafting the article or revising it critically for important intellectual content; agreed to submit to the current journal; gave final approval of the version to be published; and agree to be accountable for all aspects of the work. All the authors are eligible to be an author as per the international committee of medical journal editors (ICMJE) requirements/guidelines.

\section{ETHICAL APPROVALS}

This study does not involve experiments on animals or human subjects.

\section{REFERENCES}

1. Sudhersan C. Introduction of a multipurpose palm, Phoenix pusilla, in Kuwait. Palms 2004;48(4):191-6.

2. Envis Centre on Medicinal Plants. Available via http://envis.frlht.org/ (Accessed 23 November 2020).

3. Encyclopedia of Ayurvedic Medicinal Plants. Available via https:// www.indianmedicinalplants.info/medicinal-plants/LIST-OF-250AYURVEDIC-MEDICINAL-PLANTS.html (Accessed 23 November 2020).

4. Kumar DS, Prabhakar VS. Heart disease in ayurveda III a historical perspective. Bull Indian Inst Hist Med Hyderabad 1989;19(2):81-110.

5. Shoichet BK, McGovern SL, Wei B, Irwin JJ. Lead discovery using molecular docking. Curr Opin Chem Biol 2002;6(4):439-46; doi:10.1016/s1367-5931(02)00339-3
6. Holt PA, Chaires JB, Trent JO. Molecular docking of intercalators and groove-binders to nucleic acids using autodock and surflex. J Chem Inf Model. 2008;48(8):1602-15; doi:10.1021/ci800063v

7. Lagunin AA, Goel RK, Gawande DY, Pahwa P, Gloriozova TA, Dmitriev AV, et al. Chemo- and bioinformatics resources for in silico drug discovery from medicinal plants beyond their traditional use: a critical review. Nat Prod Rep 2014;31(11):1585-611; doi:10.1039/ c4np00068d

8. Tiwari SP, Srivastava R, Singh CS, Shukla K, Singh RK, Singh P, et al. Amylases: an overview with special reference to alpha amylase. J Global Biosci 2015;4(1):1886-1901.

9. Kumari A, Singh S. Kayastha AM. $\alpha$-amylase: general properties, mechanism and biotechnological application-a review. Curr Biotechnol 2012;1(1):98-197; doi:10.2174/2211550111201010098

10. Alqahtani AS, Hidayathulla S, Rehman MT, ElGamal AA, AlMassarani S, Razmovski-Naumovski V, et al. Alpha-amylase and alpha-glucosidase enzyme inhibition and antioxidant potential of 3-oxolupenal and katononic acid isolated from Nuxia oppositifolia. Biomolecules. 2019;10(1):1-19. doi:10.3390/biom10010061

11. Jones SA, Takeuchi T, Aletaha D, Smolen J, Choy EH, McInnes I. Interleukin 6: the biology behind the therapy. Considerations Med 2018;2:2-6; doi:10.1136/conmed-2018-000005

12. Dong Y, Dekens DW, Deyn PPD, Naude PJW, Eisel ULM. Targeting of tumor necrosis factor alpha receptors as a therapeutic strategy for neurodegenerative disorders. Antibodies 2015;4(4):369-408; doi:10.3390/antib4040369

13. Ferreira LG, Santos RND, Oliva G, Andricopulo AD. Molecular docking and structure-based drug design strategies. Molecules. 2015;20(7):13384-421; doi:10.3390/molecules200713384

14. Paramashivam SK, Elayaperumal K, Natarajan BB, Ramamoorthy $\mathrm{MD}$, Balasubramanian $\mathrm{S}$, Dhiraviam KN. In silico pharmacokinetic and molecular docking studies of small molecules derived from Indigofera aspalathoides Vahl targeting receptor tyrosine kinases. Bioinformation 2015;11(2):73-84; doi:10.6026/97320630011073

15. Murugan KK, Poojari CC, Ryavalad C, Lakshmikantha RY, Satwadi PR, Vittal RR, et al. Antidiabetic activity of endophytic fungi, penicillium species of Tabebuia argentea; in silico and experimental analysis. Res J Phytochem 2017;11(2):90-110. doi:110.3923/ rjphyto.2017.90.110

16. Ganeshpurkar A, Saluja A. In silico interaction of rutin with some immunomodulatory targets: a docking analysis. Indian J Biochem Biophys. 2018;55(2):88-94.

17. Chang WC, Wu JS, Chen CW, Kuo PL, Chien HM, Wang YT, et al. Protective effect of vanillic acid against hyperinsulinemia, hyperglycemia and hyperlipidemia via alleviating hepatic insulin resistance and inflammation in high-fat diet (HFD)-fed rats. Nutrients 2015;7(12):9946-59; doi:10.3390/nu7125514

18. Asif M, Saleem M, Yousaf S, Saadullah M, Zafar M, Khan RU, et al. Antidiabetic activity of aqueous extract of Sigesbeckia orientalis (St. Paul's Wort) in alloxan-induced diabetes model. Braz J Pharm Sci 2019;55:1-10. doi:10.1590/s2175-97902019000218408

19. Bouhlali EDT, Hmidani A, Bourkhis B, Khouya T, Ramchoun M, Filali-Zegzouti Y, et al. Phenolic profile and anti-inflammatory activity of four Moroccan date (Phoenix dactylifera $L$.) seed varieties. Heliyon 2020;6(2):1-10; doi:10.1016/j.heliyon.2020.e03436

20. Oberoi S, Kansra P. Economic menace of diabetes in India: a systematic review. Int J Diabetes Dev Ctries 2020; 40:1-12.

\section{How to cite this article:}

Saravanan V, Venkatraman A, Mohideen HS. Molecular docking of active compounds from Phoenix pusilla root extract against antidiabetic and anti-inflammatory drug targets. J Appl Biol Biotech 2021;9(S1):26-30. 